\title{
PhD defence about the ultrasound technique Vector Flow Imaging
}

Thor Bechsgaard, MD, defended the PhD thesis "In Vivo Vector Flow Imaging of Venous Flow in the Lower Extremities of healthy Volunteers and Patients with Chronic Venous Disease" on the $17^{\text {th }}$ of December. The work leading to the thesis was a collaboration between the Department of Diagnostic Radiology at University Hospital Copenhagen, Rigshospitalet and Technical University of Denmark. The PhD thesis compared the relatively new ultrasound technique with the conventional technique spectral Doppler ultrasound in healthy volunteers and patients with chronic venous disease. The technical thesis found comparable precision of the relatively new technique in all three published journal paper: "Vector and Doppler Ultrasound Velocities Evaluated in a Flow Phantom and the Femoropopliteal Vein", "Evaluation of Peak Reflux Velocities with Vector Flow Imaging and Spectral Doppler Ultrasound in Varicose Veins", and "Respiratory Variability of Peak Velocities in the Common Femoral Vein Estimated with Vector Flow Imaging and Doppler Ultrasound". The thesis is available

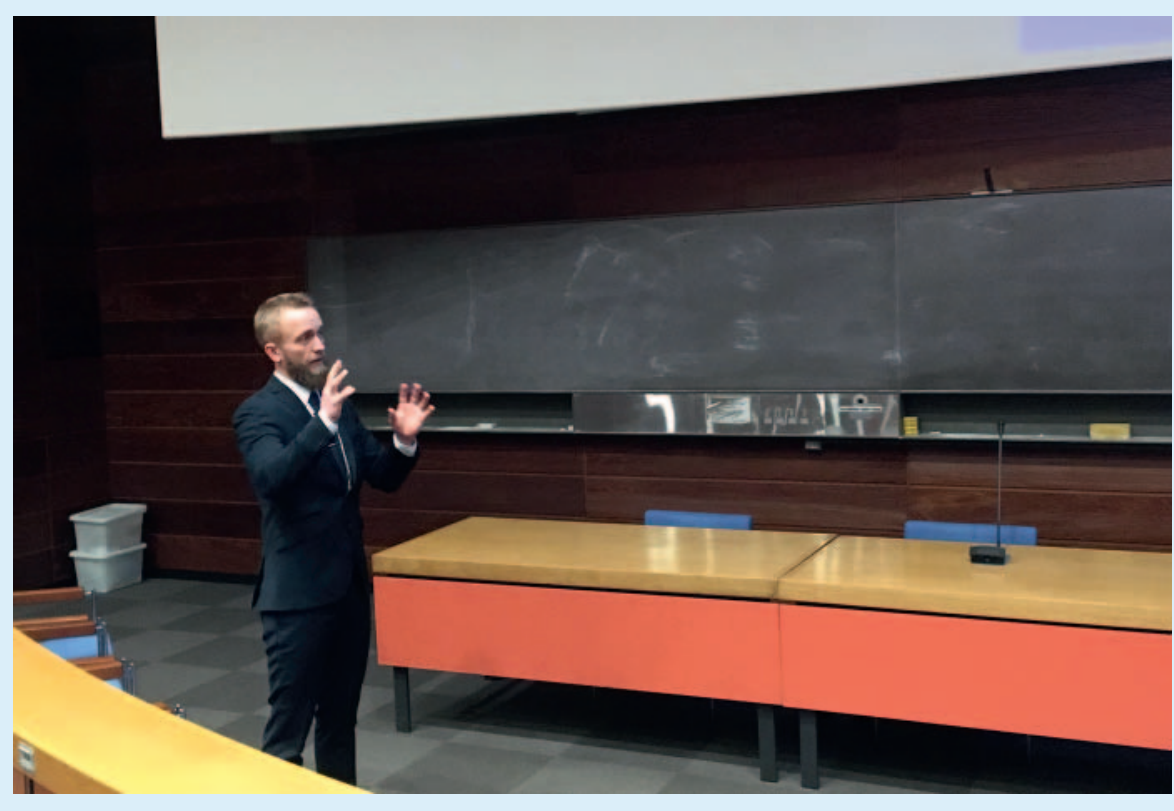

on Researchgate.com: https://www.researchgate.net/publication/330 370 583_PhD-

Healthy_Volunteers_and_Patients_with_ Chronic_Venous_Diseases. thesis_In_Vivo_Vector_Flow_Imaging_of_ Venous_Flow_in_the_Lower_Extremities_of_ 